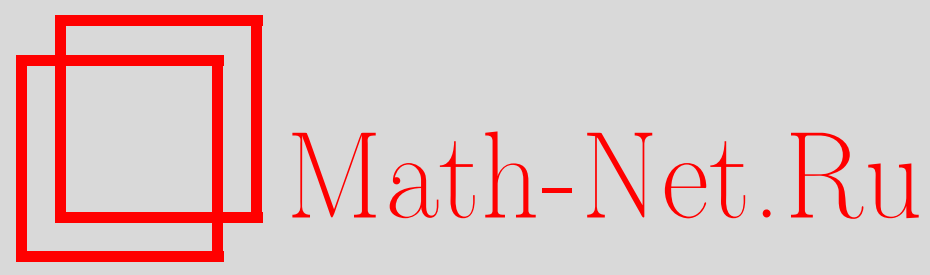

И. В. Протасов, Топологический центр полугруппы свободных ультрафильтров, Матем. заметки, 1998, том 63, выпуск $3,437-441$

DOI: https://doi.org/10.4213/mzm1300

Использование Общероссийского математического портала Math-Net.Ru подразумевает, что вы прочитали и согласны с пользовательским соглашением http://www.mathnet.ru/rus/agreement

Параметры загрузки:

IP : 54.197 .217 .227

26 апреля 2023 г., 05:38:40

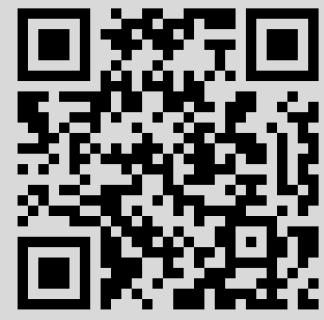




\title{
ТОПОЛОГИЧЕСКИЙ ЦЕНТР \\ ПОЛУГРУППЫ СВОБОДНЫХ УЛЬТРАФИЛЬТРОВ
}

\author{
И.В. Протасов
}

\begin{abstract}
Для любой группы $G$ доказана пустота топологического центра полугруппы свободных ультрафильтров на группе $G$.

Библиография: 4 названия.
\end{abstract}

Пусть $G$ - произвольная группа, $\beta G$ - чех-стоунова компактификация групшы $G$ как дискретного пространства. Элементами множества $\beta G$ являются всевозможные ультрафильтры на групе $G$, а базу топологии образуют подмножества $\bar{A}=\{p \in \beta G: A \in p\}$, где $p$ пробегает все подмножества групы $G$. Группа $G$ отождествляется с подмножеством всех главных ультрафильтров из $\beta G$; подмножество всех свободных ультрафильтров $\beta G \backslash G$ обозначается через $G^{*}$. Базу топологии пространства $G^{*}$ образуют множества $A^{*}=\left\{p \in G^{*}: A \in p\right\}$, где $A$ пробегает все подмножества групшы $G$.

Продолжим операцию умножения элементов групшы $G$ на множество $\beta G$. Произведение $p q$ ультрафильтров $p, q \in \beta G$ определяется указанием всех подмножеств $A \subset G$, которые являются элементами ультрафильтра $p q$

$$
A \in p q \Longleftrightarrow\left\{g \in G: A g^{-1} \in p\right\} \in q .
$$

Базу ультрафильтра $p q$ образуют подмножества группы $G$ вида $\cup\left\{P_{x} x: x \in Q\right\}$, где $Q \in q, P_{x} \in p$ для всех $x \in Q$. Операция умножения в $\beta G$ ассоциативна, а $G^{*}-$ подполугруппа полугрупшы $\beta G$. Для ультрафильтров $p, q \in \beta G$ положим $\lambda_{q}(p)=q p$, $\rho_{q}(p)=p q$. Отображение $\lambda_{q}: \beta G \rightarrow \beta G$ непрерывно для любого ультрафильтра $q \in \beta G$, а отображение $\rho_{q}: \beta G \rightarrow \beta G$ непрерывно для для любого $q \in G$.

Топологическим иентром полугруппы $\beta G$ назьвается подмножество $Z(\beta G)$ всех ультрафильтров $q \in \beta G$ таких, что отображение $\rho_{q}$ непрерывно. Обозначим через $\rho_{q}^{*}$ сужение отображения $\rho_{q}$ на подполугруппу $G^{*}$. Топологическим иентром полугруп$n ы G^{*}$ назьвается подмножество $Z\left(G^{*}\right)$ всех ультрафильтров $q \in G^{*}$ таких, что отображение $\rho_{q}^{*}$ непрерьвно.

В статье [1] доказано, что $Z(\beta G)=G$ для любой групшы $G$. В ходе доказательства этого утверждения по каждому ультрафильтру $q \in G^{*}$ подбирается ультрафильтр $p \in G^{*}$ такой, что отображение $\rho_{q}$ разрьвно в точке $p$. Естественно возникает следующий вопрос. Существует ли ультрафильтр $p \in G^{*}$ такой, что отображение $\rho_{q}$ разрывно в точке $p$ для любого ультрафильтра $q \in G^{*}$ ?

Работа выполнена при поддержке фонда INTAS, грант № 94-3420. 
В настоящей заметке доказано (теорема 1 ) существование точки $p \in G^{*}$ такой, что отображение $\rho_{q}^{*}$ разрьвно в точке $p$ для любого ультрафильтра $q \in G^{*}$ при одном лишь ограничении на бесконечную групу $G$ : мощность $|G|$ неизмерима по Уламу. Основываясь на этом утверждении, покажем (теорема 2 ), что $Z\left(G^{*}\right)=\varnothing$ для любой группы $G$.

Приведем два определения, необходимые для дальнейшего изложения. Пусть $\mathbf{k}-$ бесконечньй кардинал. Ультрафильтр $p$ на множестве $X$ назьвается k-nолны.м, если пересечение любых $\mathbf{k}$ подмножеств из $X$, которые являются элементами ультрафильтра $p$, принадлежит ультрафильтру $p$. Если свободньй ультрафильтр $p$ счетно полньй, то его дисперсионный характер $\Delta(p)=\min \{|A|: A \in p\}$ измерим по Уламу.

Элемент $x$ топологического пространства $X$ назьвается $P$-точкой, если пересечение любого счетного набора окрестностей точки $x$ также является окрестностью этой точки. Пусть $X$ - счетное дискретное пространство, $X^{*}=\beta X \backslash X, p \in X^{*}$. Ультрафильтр $p$ является $P$-точкой в пространстве $X^{*}$ тогда и только тогда, когда для любого семейства $\left\{A_{n} \in p: n<\omega\right\}$ найдется подмножество $A \in p$ такое, что подмножество $A \backslash A_{n}$ конечно для любого $n<\omega$. Вопрос о существовании $P$-точек в пространстве $X^{*}$ достаточно нетривиален. Легко построить плотное в $X^{*}$ подмножество $P$-точек, используя некоторые дополнительные к системе аксиом ZFC теоретико-множественные предположения, например, континуум-гипотезу. Однако, существуют модели ZFC, в которых пространство $X^{*}$ не имеет $P$-точек [2]. С другой стороны, пространство $X^{*}$ содержит плотное подмножество элементов, которые не являются $P$-точками.

Лемма 1. Пусть $Y=\left\{y_{n}: n<\omega\right\}-$ подмножество әруппь $G, X_{1} \subset X_{2} \subset \cdots \subset$ $X_{n} \subset \cdots$ - возрастающая иепочка конечных подмножеств из $G, X=\cup\left\{X_{n}: n<\omega\right\}$. Предположим, что $y_{n} X_{n} \cap y_{m} X_{m}=\varnothing$ дя всех $n<m$. Eсли $p, q \in \beta G, Y \in p, X \in q$ и ультрафильтр р не является $P$-точкой в пространстве $G^{*}$, то отображсение $\rho_{q}^{*}$ разрывно в точке $p$.

ДокАЗАТЕЛьство. Выберем убывающее семейство подмножеств $Y \supset V_{1} \supset \cdots \supset$ $V_{n} \supset \cdots$ групшы $G$ такое, что $V_{n} \in p, y_{1}, \ldots, y_{n} \notin V_{n}, n<\omega$, и $\cap\left\{V_{n}^{*}: n<\omega\right\}$ не является окрестностью ультрафильтра $p$ в $G^{*}$. Положим $V=\bigcup\left\{V_{n} X_{n}: n<\omega\right\}$ и заметим, что $V^{*}$ - окрестность ультрафильтра $p q$ в $G^{*}$. Допустим, что отображение $\rho_{q}^{*}$ непрерывно в точке $p$. Тогда найдется подмножество $U \in p$ такое, что $U \subset V_{1}$ и $U^{*} q \subset V^{*}$. Так как $\cap\left\{V_{n}^{*}: n<\omega\right\}$ не является окрестностью ультрафильтра $p$ в $G^{*}$, найдется номер $m$ такой, что подмножество $W=U \cap\left(V_{m} \backslash V_{m+1}\right)$ бесконечно. Зафиксируем произвольный ультрафильтр $r \in W^{*}$. Поскольку $W^{*} \subset U^{*}$, имеем $r q \in V^{*}$. Из непрерьвности умножения по второму аргументу следует, что $r Q \subset V^{*}$ для некоторого подмножества $Q \in q, Q \subset X$. Возьмем произвольный элемент $x \in Q$. Так как $V \in r x$ и $W \in r$, найдется номер $k>m$ такой, что $y_{k} \in W, y_{k} x \in V$ и $x \in X_{k}$. Поскольку $y_{k} \in V_{m} \backslash V_{m+1}$ и $y_{k} x \in y_{k} X_{k}$, то $y_{k} x \in y_{k} X_{m}$. Значит, $x \in X_{m}$ и $Q \subset X_{m}$. Получено противоречие с тем, что $Q \in q$ и $q-$ свободньй ультрафильтр.

Лемма 2. Пусть $Y, X-$ подмножества группы $G$ и $Y x \cap Y x^{\prime}=\varnothing$ для всех различных әлементов $x, x^{\prime} \in X$. Eсли $p, q \in G^{*}, X \in p, Y \in q$, ультрафильтр $p$ не является $P$-точкой в пространстве $G^{*}$, а ультрафильтр q не счетно полный, то отображение $\rho_{q}^{*}$ разрывно в точке $p$.

ДокАЗАТЕЛьство. Выберем убывающее семейство подмножеств $Y \supset V_{1} \supset \cdots \supset$ $V_{n} \supset \cdots$ такое, что $V_{n} \in p$ и $\left\{V_{n}^{*}: n<\omega\right\}$ не является окрестностью ультрафильтра $p$ в $G^{*}$. Разобьем подмножество $X$ на счетное число подмножеств $X=\bigcup\left\{X_{n}: n<\omega\right\}$ так, чтобы $X_{n} \notin q$ для всех $n<\omega$. Положим $V=\cup\left\{V_{n} X_{n}: n<\omega\right\}$ и заметим, 
что $V^{*}$ окрестность ультрафильтра $p q$ в $G^{*}$. Допустим, что отображение $\rho_{q}^{*}$ непрерывно в точке $p$. Выберем подмножество $U \in p$ такое, что $U \subset V_{1}$ и $U^{*} q \subset V^{*}$. Так как $\cap\left\{V_{n}^{*}: n<\omega\right\}$ не является окрестностью ультрафильтра $p$ в $G^{*}$, найдется номер $m$ такой, что подмножество $W=U \cap\left(V_{m} \backslash V_{m+1}\right)$ бесконечно. Зафиксируем произвольньй ультрафильтр $r \in W^{*}$. Поскольку $W^{*} \subset U^{*}$, имеем $r q \in U^{*}$. Из непрерьвности умножения по второму аргументу следует, что $r Q \subset V^{*}$ для некоторого подмножества $Q \in q$, $Q \subset X$. Возьмем произвольньй элемент $x \in Q$. Так как $V \in r x$ и $W \in r$, найдется элемент $y \in W$ такой, что $y x \in V$. Поскольку $y \in V_{m} \backslash V_{m+1}$, то $y x \in \cup\left\{V_{n} X_{n}: n \leqslant m\right\}$. Значит, $x \in \cup\left\{X_{n}: n \leqslant m\right\}$ и $Q \subset \cup\left\{X_{n}: n \leqslant m\right\}$. Получено противоречие с тем, что $X_{n} \notin q$ для всех $n<\omega$.

ЛЕмма 3. Пусть $G$ - бесконечная группа, $\mathbf{k}-$ кардинал, $p, q \in \beta G$. Операция умножения в полугруппе $\beta G$ совместно непрерывна в точке $(p, q)$, если выполняется одно из следуюших условий:

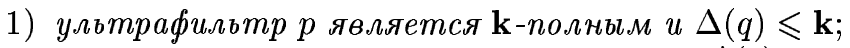

2) ультрафильтр $q$ является $\mathbf{k}-п о л н ь м ~ и ~ 2^{\Delta(p)} \leqslant \mathbf{k}$.

ДокАЗАТЕЛЬСТво. Пусть выполняется условие 1). Фиксируем произвольное подмножество $R \in p q$. Подберем подмножества $Q \in q,|Q|=\Delta(q), P_{x} \in p, x \in Q$, такие, что $\cup\left\{P_{x} x: x \in Q\right\} \subset R$. Положим $P=\cap\left\{P_{x}: x \in Q\right\}$. Так как ультрафильтр $p \mathbf{k}$-полный и $|Q| \leqslant \mathbf{k}$, то $P \in p$. Значит, $P Q \subset R$ и из [3, лемма 1.1] следует, что умножение совместно непрерьвно в точке $(p, q)$.

Пусть вьполняется условие 2). Фиксируем произвольные подмножества $R \in p q$, $P \in p,|P|=\Delta(p)$. Подберем подмножества $Q \in q, P_{x} \in p, x \in Q, P_{x} \subset P$, такие, что $\bigcup\left\{P_{x} x: x \in Q\right\} \subset R$. Разобьем множество $Q$ на классы эквивалентности, полагая $x \sim y$ тогда и только тогда, когда $P_{x}=P_{y}$. Так как число классов эквивалентности не превосходит $2^{\Delta(p)}$, а ультрафильтр $q \mathbf{k}$-полный, один из этих классов $Q^{\prime}$ является элементом ультрафильтра $q$. Положим $P^{\prime}=P_{x}, x \in Q^{\prime}$. Тогда $P^{\prime} Q^{\prime} \subset R$ и умножение совместно непрерьвно в точке $(p, q)$.

Лемма 4. Пусть $G$ - бесконечная группа, $p, q \in G^{*} . \operatorname{Ecлu} \Delta(p)=\aleph_{0}$ и ультрафильтр $р$ является $P$-точкой в пространстве $G^{*}$, то операчия умножсения $в$ полугруппе $G^{*}$ совместно непрерывна в точке $(p, q)$.

ДокАЗАТЕльство. Фиксируем произвольное подмножество $R \in p q$. Подберем подмножества $Q \in q,|Q|=\aleph_{0}, P_{x} \in p, x \in Q$, такие, что $\cup\left\{P_{x} x: x \in Q\right\} \subset R$. Так как $p-P$-точка в пространстве $G^{*}$, найдется подмножество $P \in p$ такое, что подмножество $P \backslash P_{x}$ конечно для любого $x \in Q$. Тогда $P^{*} x \in R^{*}$ для всех $x \in Q$. Значит, $P^{*} Q^{*} \subset R^{*}$.

Теорема 1. Пусть $G$ - произвольная бесконечная группа. Существует ультрафильтр $p \in G^{*}$ такой, что отображсение $\rho_{q}^{*}$ разрывно в точке $p$ для любого не счетно полного ультрафильтра $q \in G^{*}$. Мнохсество таких точек $p$ плотно в $G^{*}$.

ДокАЗАТЕЛьСтво. Предположим вначале, что группа $G$ счетна и представим ее в виде объединения возрастающей цепочки конечных подмножеств $G=\bigcup\left\{X_{n}: n<\omega\right\}$. Зафиксируем произвольное бесконечное подмножество $A \subset G$. Построим индуктивно бесконечное подмножество $Y=\left\{y_{n}: n<\omega\right\} \subset A$ такое, что $y_{n} X_{n} \cap y_{m} X_{m}=\varnothing$ для всех $n<m$. Определим ультрафильтр $p \in G^{*}$ так, что $Y \in p$ и $p$ не является $P$-точкой в $G^{*}$. По лемме 1 отображение $\rho_{q}^{*}$ разрьвно в точке $p$ для любого ультрафильтра $q \in G^{*}$.

Пусть $H$ - несчетная группа, $B$ - бесконечное подмножество из $H$. Выберем счетное подмножество $A \subset B$ и обозначим через $G$ подгруппу, порожденную подмножеством $A$. 
Как и вьше, представим $G$ в виде объединения возрастающей цепочки конечных подмножеств $G=\bigcup\left\{X_{n}: n<\omega\right\}$; определим подмножество $Y=\left\{y_{n}: n<\omega\right\}, Y \subset A$, и ультрафильтр $p \in G^{*}$.

Разложим группу $H$ на смежные классы по подгруппе $G, H=\cup\left\{G h_{\alpha}: \alpha<|H|\right\}$. Выберем убывающую цепочку подмножеств $Y \supset V_{1} \supset \cdots \supset V_{n} \supset \cdots$ так, чтобы $V_{n} \in p, n<\omega$, и $\cap\left\{V_{n}^{*}: n<\omega\right\}$ не было окрестностью ультрафильтра $p$. Положим

$$
V=\bigcup\left\{V_{n} X_{n} h_{\alpha}: n<\omega, \alpha<|H|\right\} .
$$

Возьмем произвольньй не счетно полный ультрафильтр $q \in H^{*}$ и предположим, что отображение $\rho_{q}^{*}$ непрерьвно в точке $p$. Повторяя рассуждения из доказательства леммы 1 , заключаем, что $Q \subset \cup\left\{X_{m} h_{\alpha}: \alpha<|H|\right\}$ для некоторых $Q \in q, m<\omega$. Выберем подмножество $P \in p$ такое, что $P \subset G$ и $P x \cap P y=\varnothing$ для всех различных элементов $x, y$ из конечного подмножества $X_{m}$. Так как $Q \subset \cup\left\{X_{m} h_{\alpha}: \alpha<|H|\right\}$, имеем $P x \cap P y=\varnothing$ для всех различных элементов $x, y \in Q$. Доказательство завершается применением леммы 2 .

ТЕОремА 2. Топологический иентр $Z\left(G^{*}\right)$ полугруппы $G^{*}$ пустой для любой zpynnbl $G$.

ДокАЗАТЕльСтво. Фиксируем произвольный ультрафильтр $q \in G^{*}$. Если кардинал $\Delta(q)$ неизмерим по Уламу, то по теореме $1 q \notin Z\left(G^{*}\right)$. Предположим, что кардинал $\Delta(q)$ измерим по Уламу. Выберем подгруппу $H \in q$ такую, что $|H|=\Delta(q)$. Представим подгруппу $H$ в виде объединения трансфинитной цепочки подгрупा

$$
H=\bigcup\left\{H_{\alpha}: \alpha<\Delta(q)\right\}, \quad\left|H_{\alpha}\right|<\Delta(q), \quad H_{\alpha} \subset H_{\beta}, \quad \alpha<\beta<\Delta(q) .
$$

Следуя статье [1], разобьем множество $\{\alpha: \alpha<\Delta(q)\}$ на четные и нечетные ординалы. Все предельные ординалы объявим четными, а четность ординала $\alpha+n$, где $\alpha-$ предельный ординал, определяется четностью натурального числа $n$.

Для каждого элемента $g \in H$ положим $\min g=\min \left\{\alpha: g \in H_{\alpha}\right\}$. Разобьем подгруппу $H$ на два подмножества

$$
N_{0}=\{g \in H: \min g-\text { четньй ординал }\}, \quad N_{1}=H \backslash N_{0} .
$$

Положим для определенности, что $N_{0} \in q$. Выберем ультрафильтр $p \in G^{*}$ такой, что $N_{1} \in p$ и $H_{\alpha} \notin p$ для всех $\alpha<\Delta(q)$. Ясно, что $p q \in N_{1}^{*}$. Допустим, что отображение $\rho_{q}^{*}$ непрерывно в точке $p$. Тогда найдется подмножество $P \subset N_{1}$ такое, что $P \in p$ и $P^{*} q \in N_{1}$. Возьмем произвольное счетное подмножество $R \subset P$ и фиксируем любой ультрафильтр $r \in R^{*}$. Конфинальность неизмеримого по Уламу кардинала $\Delta(q)$ несчетна. Отсюда легко следует, что $r q \in N_{0}^{*}$. Получили противоречие с тем, что $r \in R^{*}$, $R^{*} q \subset N_{1}^{*}$.

ЗАмЕчАниЕ 1. Пусть мощность $|G|$ неизмерима по Уламу. По теореме 1 множество ультрафильтров $p \in G^{*}$ таких, что отображение $\rho_{q}^{*}$ разрывно в точке $p$ для любого ультрафильтра $q \in G^{*}$, плотно в $G^{*}$. Из леммы 3 следует, что это утверждение неверно, если мощность $|G|$ измерима по Уламу.

Вопрос 1. Пусть мощность $|G|$ измерима по Уламу. Существует ли ультрафильтр $p \in G^{*}$ такой, что отображение $\rho_{q}^{*}$ разрывно в точке $p$ для любого ультрафильтра $q \in G^{*} ?$ 
ЗАмЕчАниЕ 2 . Пусть $G$ - произвольная группа, $p \in \beta G$. Если отображение $\rho_{q}$ непрерьвно в точке $p$ для любого ультрафильтра $q \in G^{*}$, то по теореме 3.5 из [1] $p \in G$. Если группа $G$ счетна и $p-P$-точка в пространстве $G^{*}$, то по лемме 4 отображение $\rho_{q}^{*}$ непрерьвно в точке $p$ для любого ультрафильтра $q \in G^{*}$.

Вопрос 2 . Пусть $G$ - счетная група, $p \in G^{*}$ и отображение $\rho_{q}^{*}$ непрерьвно в точке $p$ для любого ультрафильтра $q \in G^{*}$. Верно ли, что $p-P$-точка в пространстве $G^{*} ?$

ЗАмЕчАнИЕ 3 . В связи с теоремой 1 и замечанием 2 возникает следуюший вопрос. Пусть свободньй ультрафильтр $p$ на счетной групе $G$ не является $P$-точкой в пространстве $G^{*}$. Верно ли, что отображение $\rho_{q}^{*}$ разрьвно в точке $p$ для любого ультрафильтра $q \in G^{*} ?$ При некоторых естественных теоретико-множественных предположениях ответ на этот вопрос отрицательньй.

ПРИмЕР. Пусть $B$ - счетная группа экспоненты $2, p$ - сильно суммируемый ультрафильтр из $B^{*}$. По определению [4] для каждого подмножества $P \in p$ найдется подгруппа $H$ такая, что $H \backslash\{e\} \subset P, H \in p$, где $e$ - единица группы $B$. Очевидно, что отображение $\rho_{p}$ непрерьвно в точке $p$. Поскольку $p$ - идемпотент полугрупшы $B^{*}, p$ не является $P$-точкой в $B^{*}$.

Выберем произвольную подгруппу бесконечного индекса $Y \subset B$ так, что $Y \in p$. По лемме 2 найдется ультрафильтр $q \in B^{*}$ такой, что отображение $\rho_{q}^{*}$ разрьвно в точке $p$.

Этот же пример и теорема 4.2 из [3] показывают, что из непрерьвности отображения $\rho_{p}$ в точке $p$ не следует совместная непрерьвность умножения в полугрупе $\beta G$ в точке $(p, p)$.

Сильно суммируемые ультрафильтры на группе $B$ можно построить, например, с помощью аксиомы Мартина. В статье [4] доказано, что существование сильно суммируемого ультрафильтра на групе $B$ влечет существование $P$-точки в пространстве $\mathbb{N}^{*}$, где $\mathbb{N}$ - дискретное пространство натуральных чисел.

Вопрос 3. Предположим, что на некоторой счетной групше найдутся свободные ультрафильтры $p, q$ такие, что отображение $\rho_{q}^{*}$ непрерьвно в точке $p$. Следует ли из этого предположения существование $P$-точки в пространстве $\mathbb{N}^{*}$ ?

Автор признателен профессору Дж. Пиму, предоставившему рукопись статьи [1] до ее опубликования.

\section{СПИСОК ЦИТИРОВАННОЙ ЛИТЕРАТУРЫ}

[1] Lau A., Pym J. The topological centre of a compactification of a locally compact group // Math. Z. 1995. V. 219. P. 567-579.

[2] Shelah S. Proper Forcing. Lecture Notes in Math. V. 940. Berlin: Springer, 1982.

[3] Протасов И.В. Точки совместной непрерывности полугруппы ультрафильтров абелевой группы // Матем. сб. 1996. Т. 187. № 2. С. 131-140.

[4] Blass A., Hindman N. On strongly summable and union ultrafilters // Trans. Amer. Math. Soc. 1987. V. 304. № 1. P. 83-99.

Киевский государственный университет им. Т. Шевченко 